\title{
Emotion Regulatory Brain Function and SSRI Treatment in PTSD: Neural Correlates and Predictors of Change
}

\author{
Annmarie MacNamara ${ }^{*, 1}$, Christine A Rabinak ${ }^{2,3}$, Amy E Kennedy 1,4, Daniel A Fitzgerald ${ }^{1,4}$, Israel Liberzon ${ }^{5,6}$, \\ Murray B Stein ${ }^{7,8,9}$ and K Luan Phan ${ }^{1,4,10,11}$
}

'Department of Psychiatry, University of Illinois at Chicago, Chicago, IL, USA; ' ${ }^{2}$ Department of Pharmacy Practice, Wayne State University, Detroit, MI, USA; ${ }^{3}$ Department of Psychiatry and Behavioral Neurosciences, Wayne State University, Detroit, MI, USA; ${ }^{4}$ Mental Health Service Line, Jesse Brown VA Medical Center, Chicago, IL, USA; ${ }^{5}$ Department of Psychiatry, University of Michigan and Mental Health Service, VA Ann Arbor Healthcare System, Ann Arbor, MI, USA; ${ }^{6}$ Department of Psychology, University of Michigan, Ann Arbor, MI, USA; ${ }^{7}$ Department of Psychiatry, University of California at San Diego, San Diego, CA, USA; ${ }^{8}$ Department of Family Medicine and Public Health, University of California at San Diego, San Diego, CA, USA; ${ }^{9}$ VA San Diego Healthcare System, San Diego, CA, USA; ${ }^{10}$ Department of Psychology and the Graduate Program in Neuroscience, University of Illinois at Chicago, Chicago, IL, USA; "'Department of Anatomy and Cell Biology, University of Illinois at Chicago, Chicago, IL, USA

\begin{abstract}
Posttraumatic stress disorder (PTSD) — a chronic, debilitating condition, broadly characterized by emotion dysregulation —is prevalent among US military personnel who have returned from Operations Enduring Freedom (OEF) and Iraqi Freedom (OIF). Selective serotonin reuptake inhibitors (SSRIs) are a first-line treatment for PTSD, but treatment mechanisms are unknown and patient response varies. SSRIs may exert their effects by remediating emotion regulatory brain activity and individual differences in patient response might be explained, in part, by pre-treatment differences in neural systems supporting the downregulation of negative affect. Thirty-four OEF/OIF veterans, 17 with PTSD and 17 without PTSD underwent 2 functional magnetic resonance imaging scans 12 weeks apart. At each scan, they performed an emotion regulation task; in the interim, veterans with PTSD were treated with the SSRI, paroxetine. SSRI treatment increased activation in both the left dorsolateral prefrontal cortex (PFC) and supplementary motor area (SMA) during emotion regulation, although only change in the SMA over time occurred in veterans with PTSD and not those without PTSD. Less activation of the right ventrolateral PFC/ inferior frontal gyrus during pre-treatment emotion regulation was associated with greater reduction in PTSD symptoms with SSRI treatment, irrespective of pre-treatment severity. Patients with the least recruitment of prefrontal emotion regulatory brain regions may benefit most from treatment with SSRIs, which appear to augment activity in these regions.

Neuropsychopharmacology (2016) 4I, 6II-6I8; doi:10.1038/npp.2015.190; published online 5 August 2015
\end{abstract}

\section{INTRODUCTION}

Posttraumatic stress disorder (PTSD) is a chronic condition characterized by intrusive memories, hyperarousal, and severe deficits in negative affect regulation (APA, 2000). Among US military personnel who have returned from overseas deployment, rates of PTSD are especially high; for instance, of the 2.2 million US soldiers deployed in Operations Enduring Freedom (OEF), Iraqi Freedom (OIF) and New Dawn (IOM, 2013), 14\%-16\% have developed PTSD (Hoge et al, 2004). Selective serotonin reuptake inhibitors (SSRIs) are an evidence-based pharmacological treatment for PTSD; however, treatment mechanisms are not fully understood and individual patients differ in therapeutic response. For instance,

\footnotetext{
*Correspondence: Dr A MacNamara, Department of Psychiatry, University of Illinois at Chicago, 1747 West Roosevelt Road, Chicago, IL 60608, USA, Tel: + I 312413 4707, Fax: + I 312413 1703,

E-mail: amacnamara@psych.uic.edu

Received 5 February 2015; revised 17 June 2015; accepted 19 June 2015; accepted article preview online 26 June 2015
}

only about $60 \%$ of PTSD patients respond to treatment with SSRIs, with just $20 \%-30 \%$ of those in treatment achieving complete remission of symptoms (Berger et al, 2009). Neural measures of emotion regulatory function may be useful in determining mechanisms of treatment change and in identifying predictors of treatment outcome.

Among psychiatrically healthy individuals, emotion regulation is known to engage prefrontal brain regions such as the dorsolateral prefrontal cortex (dlPFC), the dorsomedial PFC, the ventrolateral PFC (vlPFC), and the ventromedial PFC (vmPFC; Buhle et al, 2013; Frank et al, 2014). These regions are implicated in PTSD pathophysiology such that individuals with PTSD exhibit reduced activation in the lateral PFC (Rabinak et al, 2014; Vermetten et al, 2007), vmPFC (Bremner et al, 1999), and other frontal areas during the provocation of anxious states and negative affect. In addition, some studies have found evidence of increased amygdala reactivity to negative stimuli in individuals with PTSD (eg, Shin et al, 2004, but see Phan et al, 2006). Thus, the overall pattern of effects observed for PTSD seems to be reduced recruitment of 
prefrontal emotion regulatory regions, with some evidence for increased activity in limbic regions (Hayes et al, 2012; Simmons and Matthews, 2012).

In two recent studies, New et al (2009) and Rabinak et al (2014) examined individuals with PTSD during volitional attempts to reduce their negative affect evoked by aversive images using cognitive reappraisal, a highly effective emotion regulation strategy that involves changing the meaning of emotional stimuli (Gross, 1998). New et al (2009) found that females with PTSD related to a sexual trauma showed reduced recruitment of the precentral gyrus/lateral PFC and the supplementary motor area (SMA) during reappraisal of negative affect. In the Rabinak et al (2014) study, OEF/OIF veterans suffering from military-related PTSD showed reduced recruitment of the dlPFC during reappraisal of negative affect. Therefore, results from these studies converge to suggest that individuals with PTSD may be characterized by deficient recruitment of lateral prefrontal regions critical for cognitive control and negative affect regulation (Buhle et al, 2013).

SSRI treatment may in part ameliorate negative affect regulation difficulties in PTSD (Robert et al, 2006; Seedat et al, 2002). Moreover, work conducted in depressed patients has suggested that treatment with SSRIs may increase activation in the lateral PFC (Ma, 2014). Despite these observations, no study to date has examined whether SSRI treatment for PTSD might work by enhancing brain emotion regulatory function or whether individual differences in SSRI treatment response may be explained, in part, by brain emotion regulatory function before treatment. One prior study in PTSD found that 8 weeks of treatment with SSRI, citalopram, reduced resting state activation in the left medial temporal cortex, whereas greater activation in the medial PFC (left paracingulate cortex) post-treatment was found to be associated with greater symptom reduction (Seedat et al, 2004). A second study reported that a single PTSD patient showed normalization of activation in the insula, PFC, inferior frontal gyrus (IFG), cerebellum, precuneus and SMA following treatment with the SSRI, fluoxetine (Fernandez et al, 2001). Results from these studies are consistent with the idea that treatment with SSRIs may restore functioning in the PFC. Importantly, however, results differ somewhat between studies (ie, subregion of the PFC involved), neither study used a task designed to probe emotion regulatory brain function, and predictors of treatment outcome (ie, at baseline) were not identified.

The current study reports on OEF/OIF veterans with PTSD, who received an initial scan (Rabinak et al, 2014), followed by 12 weeks of treatment with the SSRI, paroxetine; all participants (both with and without PTSD) were invited to return for a second scan 12 weeks later, in which they performed the same reappraisal task. We hypothesized that SSRI treatment would increase reappraisal-related activation in the dlPFC, found to be deficient in OEF/OIF veterans with PTSD at baseline (Rabinak et al, 2014). Based on the findings observed in depression, we thought it possible that either less (Miller et al, 2013) or more (Fu et al, 2004) pre-treatment activation in reappraisal-related prefrontal regions (ie,precentral gyrus, lateral (dlPFC and vlPFC) and medial PFC, angular gyrus, IFG, and SMA; Kohn et al, 2014) would correspond to greater benefits from treatment with an SSRI. Rabinak et al (2014) did not observe $M>R$ group differences at pre-treatment in the amygdala. Therefore, we hypothesized that treatment would not affect $M>R$ amygdala activity.

\section{MATERIALS AND METHODS}

\section{Participants}

A detailed description of inclusion and exclusion criteria and recruitment procedures can be found in Rabinak et al (2014). In brief, 64 right-handed OEF/OIF male veterans were initially enrolled in the study (36 treatment-seeking veterans with a diagnosis of PTSD and 28 non-treatment-seeking veterans who had been exposed to combat trauma but were free from PTSD-combat-exposed controls (CECs)). Of these, 20 participants with PTSD completed a full course of SSRI treatment and 17 of these returned for a second scan and had useable functional magnetic resonance imaging (fMRI) data at both pre- and post-treatment (eg, $<3 \mathrm{~mm}$ of head motion); 20 CEC participants completed the study and 17 of these had useable fMRI data for both scans (eg, $<3 \mathrm{~mm}$ of head motion). Therefore, the final sample consisted of 17 veterans in the PTSD group and 17 in the CEC group. Participant characteristics are presented in Table 1. Compared with the CEC group, the PTSD group was younger $(t(32)=2.08, p=0.05)$ and had fewer years of education $(t(32)=5.17, p<0.001)$.

Psychiatric diagnoses were established using the Structured Clinical Interview for Diagnostic and Statistical Manual of Mental Disorders, DSM-IV (SCID-NP; First et al, 2002). Psychiatric comorbidities at the initial scanning in the PTSD group were $n=2$ major depressive disorder and $n=2$ alcohol abuse (when the two participants in the PTSD group who met criteria for current alcohol abuse were excluded, fMRI results did not change substantially); in the CEC group, $n=1$ generalized anxiety disorder. Dimensional clinical measures included the Clinician-Administered PTSD Scale (CAPS; Blake et al, 1995), the PTSD Checklist: Military (PCL-M; Blanchard et al, 1996), the Combat Exposure Scale (CES; Keane et al, 1989), the Hamilton Anxiety Scale (HAMA; Maier et al, 1988), the Hamilton Depression Inventory (HAM-D; Hamilton, 1960), and the Beck Depression Inventory (BDI-II; Beck et al, 1996). The PTSD group reported higher scores on the CAPS $(t(23.22)=19.93, p<0.001)$, PCL-M $\quad(t(32)=11.39, p<0.001)$, HAM-A $(t(26.39)=8.87$, $p<0.001)$, HAM-D $(t(26.71)=8.09, p<0.001)$, BDI $(t(32)=$ $7.48, p<0.001)$, and CES $(t(32)=2.12, p<0.05)$ scales compared with the CEC group (when one CEC participant was removed from analyses, groups did not significantly differ on age and CES scores, and fMRI results did not change substantially).

Exclusion criteria for PTSD participants were as follows: (1) a history of bipolar disorder, psychotic disorder, mental retardation, or developmental disorders; (2) current psychotherapy treatment of any kind; (3) intolerance or contraindication to taking paroxetine or another SSRI, and (4) previous failure to respond to SSRI treatment (for PTSD or another disorder). Combat control subjects were excluded if they had a history of PTSD or partial/subthreshold PTSD preceding their most recent deployment. None of the subjects had a history of a major medical or neurological illness, or a history of traumatic brain injury. All subjects were right-handed and were required to be free from psychotropic medications for at least 8 weeks at the time 
Table I Clinical and Demographic Characteristics of Participants

\begin{tabular}{|c|c|c|c|c|c|c|}
\hline & \multicolumn{2}{|c|}{$\operatorname{CEC}(n=17)$} & \multicolumn{4}{|c|}{ PTSD $(n=17)$} \\
\hline & $\mathbf{M}$ & SD & \multicolumn{2}{|c|}{ M } & \multicolumn{2}{|c|}{ SD } \\
\hline Age & 35.35 & 9.79 & \multicolumn{2}{|c|}{29.53} & \multicolumn{2}{|c|}{6.16} \\
\hline Years of education & 15.59 & 1.54 & \multicolumn{2}{|c|}{13.06} & \multicolumn{2}{|c|}{1.30} \\
\hline \multirow[t]{2}{*}{ Combat exposure scale } & 20.29 & 4.38 & \multicolumn{2}{|c|}{$\begin{array}{c}24.18 \\
\text { Pre-treatment }\end{array}$} & \multicolumn{2}{|c|}{6.17} \\
\hline & & & $\mathbf{M}$ & SD & $\mathbf{M}$ & SD \\
\hline Beck depression inventory & 4.94 & 6.26 & 22.47 & 7.36 & 8.71 & 6.44 \\
\hline Hamilton depression & 1.65 & 2.29 & 10.18 & 3.70 & 6.06 & 4.51 \\
\hline Hamilton anxiety & 2.23 & 2.51 & 12.65 & 4.14 & 6.71 & 4.98 \\
\hline
\end{tabular}

Abbreviations: CEC, combat-exposed control; GAF, global assessment of functioning; M, mean; PTSD, posttraumatic stress disorder.

of study entry, with negative urine toxicology screens at each scan. Study procedures were in compliance with the Helsinki Declaration of 1975 (as revised in 1983) and were approved by the University of Michigan and VA Ann Arbor Healthcare System institutional review boards.

\section{SSRI Paroxetine Treatment}

Treatment for PTSD consisted of administration of the SSRI, paroxetine, in an open-label, fixed-dosing design over 12 weeks. The study psychiatrist (KLP) assessed patients at $1,2,4,8$, and 12 weeks, to evaluate symptom change and adverse events, with the target dose of $20 \mathrm{mg}$ /day reached at 2 weeks. Although blood was not taken to assess paroxetine levels, the psychiatrist inquired about missed pills and pill counts were performed. No subject reported missing more than two consecutive doses throughout the entire study and no subject regularly (more than 3 times) failed to take the medication. At 8 weeks, medication dosage was increased to $40 \mathrm{mg} /$ day (maximum dose) if the subject failed to show improvement using the Clinical Global Impression-Improvement scale (ie, CGI-I score $>2$ ). At study completion, all subjects were on stable doses of paroxetine for at least 4 weeks before their final fMRI scan at 12 weeks. Median dosage of paroxetine at 12 weeks was $20 \mathrm{mg}$.

\section{Cognitive Reappraisal Task}

A detailed description of the task is provided in Rabinak et al (2014). In brief, there were 64 unpleasant and 32 neutral International Affective Picture System images (Lang et al, 2008). In the 'Look' condition, participants viewed neutral pictures. In the 'Maintain' condition, participants viewed unpleasant pictures and were instructed to experience these images naturally. During the 'Reappraise' condition, participants viewed unpleasant pictures and were instructed to use the cognitive strategy of reappraisal to reduce negative affect. Before beginning the experiment, participants were trained in the strategy of cognitive reappraisal (Ochsner et al, 2002; Phan et al, 2005).

There were eight 20-s blocks of each condition (comprising four images presented for $5 \mathrm{~s}$ each), which were interspersed with 20-s baseline blocks (comprising a white fixation cross presented on a black background). At the beginning of each block, the word 'Look', 'Maintain,' or 'Reappraise' appeared in white text on a black screen for $5 \mathrm{~s}$. Block order was pseudo-randomized over four separate runs of $5 \mathrm{~min}$ each.

\section{Image Acquisition}

fMRI based on BOLD contrast was performed on a 3T GE Signa System (General Electric, Milwaukee, WI) using a standard radiofrequency coil at the University of Michigan Functional MRI Laboratory. A standard T2*-sensitive gradient-echo reverse spiral sequence was used $(2 \mathrm{~s} \mathrm{TR}$, $30 \mathrm{~ms}$ TE, $90^{\circ} \mathrm{flip}, 64 \times 64$ matrix, $22 \mathrm{~cm}$ FOV , 43 axial, $3 \mathrm{~mm}$ slices, 600 volumes). Data from all participants included in the analyses met our criteria for image quality with minimal motion correction (all movements $\leq 3 \mathrm{~mm}$ in any direction across functional runs).

\section{fMRI Data Analysis}

The first four volumes from each run were discarded to allow for the magnetization to reach equilibrium. Statistical Parametric Mapping (SPM 8) software (Wellcome Trust Centre for Neuroimaging, London, www.fil.ion.ucl.ac.uk/ spm) was used to perform conventional preprocessing steps. In brief, slice-time correction was performed to account for temporal differences between slice collection order, images were spatially realigned to the first image of the first run, functional images were normalized to a Montreal Neurological Institute $(\mathrm{MNI})$ template using the EPI template, resampled to $2 \mathrm{~mm}^{3}$ voxels, and smoothed with an $8-\mathrm{mm}$ isotropic Gaussian kernel. 
The time-series data were subjected to a general linear model, convolved with the canonical hemodynamic response function, and filtered with a 128-s high-pass filter. Conditions of interest were the Reappraise and Maintain trials, which were modeled separately, with effects estimated for each voxel for each participant. Following processing at the first-level, Reappraise $>$ Maintain $(R>M)$ contrasts, created separately for each scan, were taken to the second level for randomeffects analysis; Rabinak et al (2014) validated the ERT showing that both CEC and PTSD groups activated dorsal and lateral PFC, IFG, and SMA during reappraisal $(\mathrm{R}>\mathrm{M})$, consistent with recent meta-analyses of reappraisal-based emotion regulation (Buhle et al, 2013; Kohn et al, 2014).

Group differences were not examined in the present analysis but are reported in prior work (Rabinak et al, 2014). At the second level, we performed two sets of analyses. First, to examine overall neural correlates of treatment in the PTSD group, we assessed $\mathrm{R}>\mathrm{M}$ brain activity elicited after treatment ('Post') compared with before treatment ('Pre') using a paired $t$-test. To determine whether Post $>$ Pre change was specific to the PTSD group, we extracted $\mathrm{R}>\mathrm{M} \quad \beta$ weights from each area of activation and performed a 2 (Group: CEC, PTSD) $\times 2$ (Time: Pre, Post) mixed-measures analysis of variance (ANOVA). Second, we examined whole-brain $\mathrm{R}>\mathrm{M}$ brain activity before treatment that covaried with individual differences in treatment response (PreCAPS $>$ PostCAPS score), controlling for baseline symptomatology (PreCAPS score). That is, participants' Pre $\mathrm{R}>\mathrm{M}$ hemodynamic activity was regressed onto participants' Pre $>$ PostCAPS score change, controlling for PreCAPS.

For both analyses, we used a region-of-interest (ROI) approach to test our a priori hypotheses that treatment would increase activity in emotion regulatory prefrontal brain regions. For these analyses, precentral gyrus, lateral PFC, IFG, SMA, and inferior parietal and angular gyri-all regions considered bilaterally-comprised the ROI partialbrain mask (volume $=290 \mathrm{~cm}^{3}$ ) and were chosen based on an up-to-date meta-analysis of fMRI studies of emotion regulation (Kohn et al, 2014). An ROI mask encompassing coordinates reported by Kohn et al (2014) was created using anatomical landmarks taken from the automated anatomical labeling atlas (Tzourio-Mazoyer et al, 2002). Clusters of activation were initially identified using an uncorrected voxel threshold of $p<0.005$ and then subjected to correction for multiple comparisons within the ROI mask as determined via simulation using the $3 \mathrm{dClustSim}$ utility (10000 iterations; http://afni.nimh.nih.gov/pub/dist/doc/program_help/3dClust Sim.html). Given smoothness estimates of the data within the mask, a family-wise error correction at $\alpha<0.05$ was realized using a voxel threshold of $p<0.005$ with minimum cluster size of 96 voxels (volume $=768 \mathrm{~mm}^{3}$ ) for the Post $>$ Pre analysis and using a minimum cluster size of 125 voxels (volume $=1000$ $\mathrm{mm}^{3}$ ) for the analysis examining predictors of treatment-related symptom change. In addition to this ROI mask, we examined treatment effects using anatomically defined ROIs for each of the left and right amygdala, and a $p$-value corrected for multiple comparisons (ie, small-volume correction). For both analyses, we also examined whole-brain activation that occurred outside these a priori $\mathrm{ROI}$ regions and surpassed a threshold of $p<0.05$, FDR corrected; however, no additional clusters of activation surpassed this threshold and thus are not reported here. To clarify the direction of significant results from each analysis, we extracted BOLD signal responses (parameter estimates, $\beta$-weights in arbitrary units of activation) averaged across all voxels within a $10-\mathrm{mm}$ radius sphere surrounding the peak maxima voxel.

\section{RESULTS}

\section{Treatment Effect on PTSD Symptoms}

Twelve weeks of paroxetine treatment was effective in reducing PTSD symptomatology, as indexed by the CAPS $($ Pre Mean $(M)=67.94$, Standard Deviation $(S D)=11.64$; Post $M=26.59, \mathrm{SD}=16.33 ; t(16)=12.57, p<0.001)$. Posttreatment, more than two-thirds of the sample (12 out of 17) showed at least a 50\% reduction in their CAPS scores.

\section{fMRI BOLD Response}

Treatment effects. First, we examined the Post $(\mathrm{R}>\mathrm{M})$ $>$ Pre $(\mathrm{R}>\mathrm{M})$ contrast within the PTSD group, to determine whether SSRI treatment effectively increased emotion regulatory brain activity. Results showed that compared with pre-treatment, individuals with PTSD showed greater $\mathrm{R}>\mathrm{M}$ activation following treatment in the left dlPFC (peak MNI coordinate, $-44,18,28 ; 1104 \mathrm{~mm}^{3} ; Z=3.77, p<0.05$, corrected; Figure 1a) and in the SMA (peak MNI coordinate, 4 , 14,$70 ; 1624 \mathrm{~mm}^{3} ; Z=3.37, p<0.05$, corrected; Figure 2a). No other regional brain activity surpassed our cluster size threshold corrected for multiple comparisons. Follow-up inspection of ROI-extracted BOLD signal ( $\beta$ weights) from the left dlPFC and SMA confirmed the direction of increased Post $>$ Pre dlPFC and SMA activation during R $>M$ trials (left dlPFC: Pre R $>M$, $M=0.44, \mathrm{SD}=0.97$ and Post $\mathrm{R}>\mathrm{M}, M=1.02, \mathrm{SD}=1.01$, Figure 1b; SMA: Pre $\mathrm{R}>\mathrm{M}, M=0.28, \mathrm{SD}=1.01$ and Post $\mathrm{R}>\mathrm{M}, M=1.35, \mathrm{SD}=1.39$, Figure $2 \mathrm{~b}$ ). There was also a trend for Pre to Post change in the left dlPFC to correlate with symptom change (Pearson's $r(15)=0.47, p=0.06$ ), such that greater change in this region corresponded to greater improvement with treatment; the same correlation did not approach significance for the SMA (Pearson's $r(15)=0.22, p=0.40$ ).

Using extracted $\beta$-weights, we failed to observe a significant Group $\times$ Time interaction for the left dlPFC $(p>0.14)$. Nevertheless, we found that CEC participants failed to show a change in $\mathrm{R}>\mathrm{M}$ activation in the left dlPFC over the 12-week period (Pre $\mathrm{R}>\mathrm{M}, M=1.02, \mathrm{SD}=1.44$ and Post $\mathrm{R}>\mathrm{M}$, $M=0.95, \mathrm{SD}=1.29, t(16)=0.17, p=0.87$, Figure $1 \mathrm{~b})$. We did observe a significant Group $\times$ Time interaction for the SMA: $F$ $(1,32)=5.28, p=0.03, \eta_{\mathrm{p}}^{2}=0.14$. CEC participants failed to show a change in $\mathrm{R}>\mathrm{M}$ activation in the SMA over the 12-week period (Pre $\mathrm{R}>\mathrm{M}, M=1.09, \mathrm{SD}=2.31$ and Post $\mathrm{R}>\mathrm{M}, M=0.72, \mathrm{SD}=1.62, t(16)=0.69, p=0.50$, Figure $2 \mathrm{~b}$ ) (Using a whole-brain, 2 (Group: CEC, PTSD) $\times 2$ (Time: Pre, Post) mixed-measures ANOVA performed on $\mathrm{R}>\mathrm{M}$ activation, we did not observe clusters of activation that surpassed our AlphaSim or FDR $p<0.05$ whole-brain thresholds for the Group $\times$ Time interaction.).

Treatment-related modulation of $\mathrm{M}>\mathrm{R}$ amygdala activity was not observed (data not shown).

Predictors of symptom change. There was a negative correlation between Pre-Post symptom change and pre-treatment $\mathrm{R}>\mathrm{M}$ activity in the right vlPFC/IFG (peak MNI coordinate, 
a

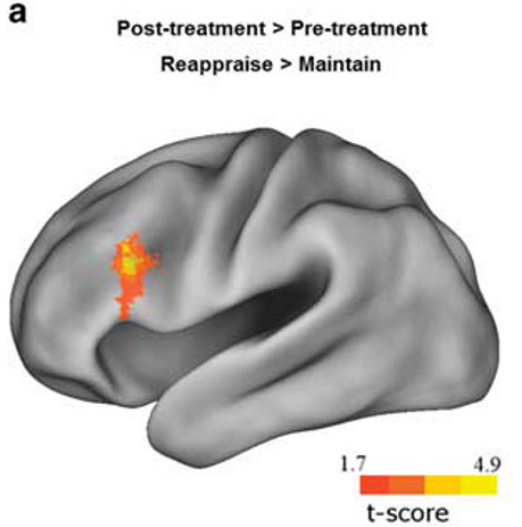

b

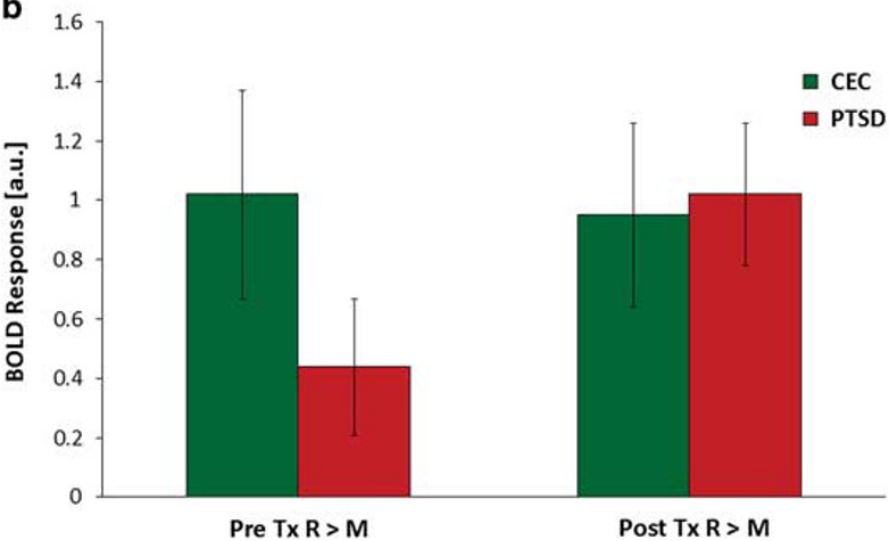

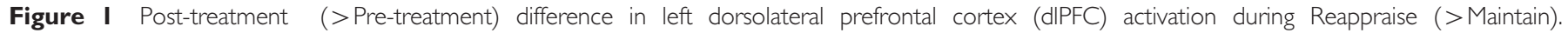
(a) One-sample voxel-wise statistical t-map overlaid on a canonical brain rendering (Montreal Neurological Institute (MNI) sagittal), showing increased

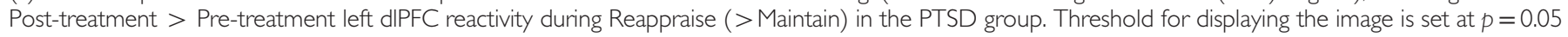

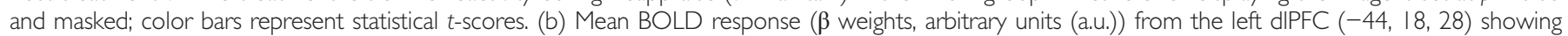

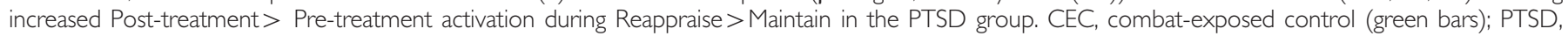
posttraumatic stress disorder (red bars); R>M, Reappraise > Maintain; Tx, Treatment. Error bars indicate SEM.
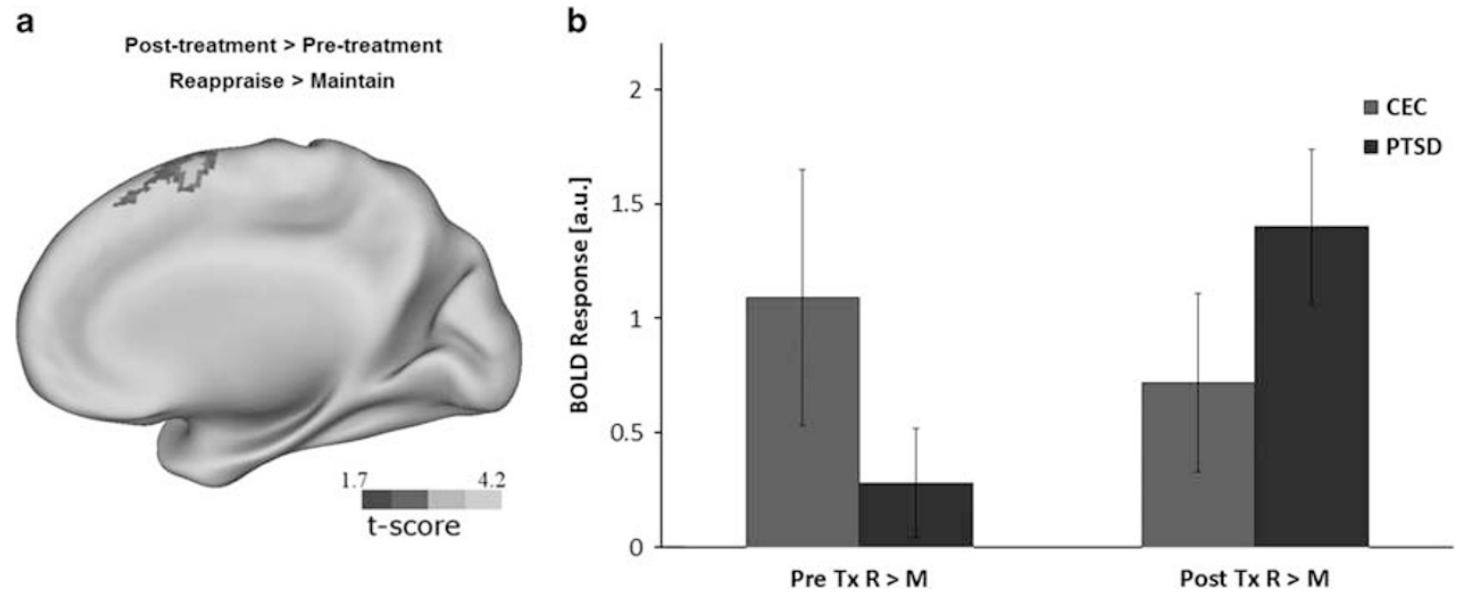

Figure 2 Post-treatment (>Pre-treatment) difference in supplementary motor area (SMA) activation during Reappraise ( $>$ Maintain). (a) One-sample voxel-wise statistical $t$-map overlaid on a canonical brain rendering (Montreal Neurological Institute (MNI) sagittal), showing increased Post-treatment $>$ Pretreatment SMA reactivity during Reappraise (> Maintain) in the PTSD group. Threshold for displaying the image is set at $p=0.05$ and masked; color bars represent statistical t-scores. (b) Mean BOLD response ( $\beta$ weights, arbitrary units (a.u.)) from the SMA (4, 14, 70) showing increased Post-treatment $>$ Pretreatment activation during Reappraise > Maintain in the PTSD group. CEC, combat-exposed control (green bars); PTSD, posttraumatic stress disorder (red bars); R>M, Reappraise > Maintain; Tx, Treatment. Error bars indicate SEM.

$46, \quad 44,-10 ; 2152 \mathrm{~mm}^{3} ; \quad Z=3.96, \quad p<0.05$, corrected, Figure 3a). Participants with less pre-treatment $\mathrm{R}>\mathrm{M}$ activity in the right vlPFC/IFG showed greater treatment-related gains (PTSD symptom reduction) (Figure $3 \mathrm{~b}$ ). No other regional brain activity surpassed our cluster size threshold for correction for multiple comparisons.

\section{DISCUSSION}

The current study examined neural correlates and predictors of treatment change in the context of a 12-week clinical trial with the SSRI paroxetine in OEF/OIF veterans with PTSD. Treatment with SSRIs increased reappraisal-related activation in the left dlPFC and SMA. In addition, lower pretreatment reappraisal-related right vlPFC/IFG activation was predictive of increased treatment gain, controlling for symptom severity before treatment. These findings suggest that PTSD patients with the least emotion regulatory brain function may stand to gain the most from SSRIs, which appear to normalize BOLD response in prefrontal regions during reappraisal.

The dlPFC is implicated in domain-general cognitive control (Miller and Cohen, 2001) and plays a key role in the initiation and maintenance of emotion regulation (eg, Kohn et al, 2014). However, as the dlPFC is not directly connected to subcortical emotion-processing regions such as the amygdala (Ghashghaei et al, 2007), it likely exerts its effects on these regions indirectly. A recent meta-analysis suggested that the SMA, which is believed to be among the network of neural regions mediating top-down control of negative affect (Ray and Zald, 2012) may be involved in implementing dlPFC-initiated emotion regulatory effects (Kohn et al, 2014). The SMA is involved in 
a

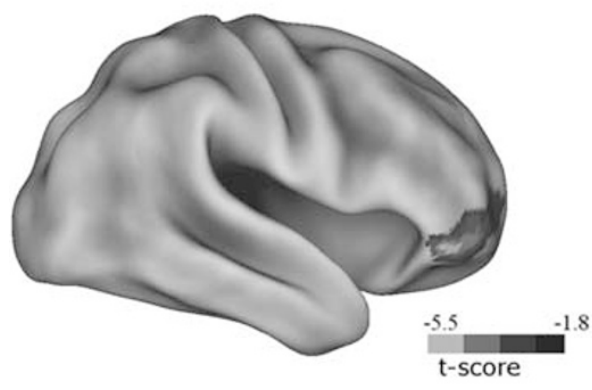

b

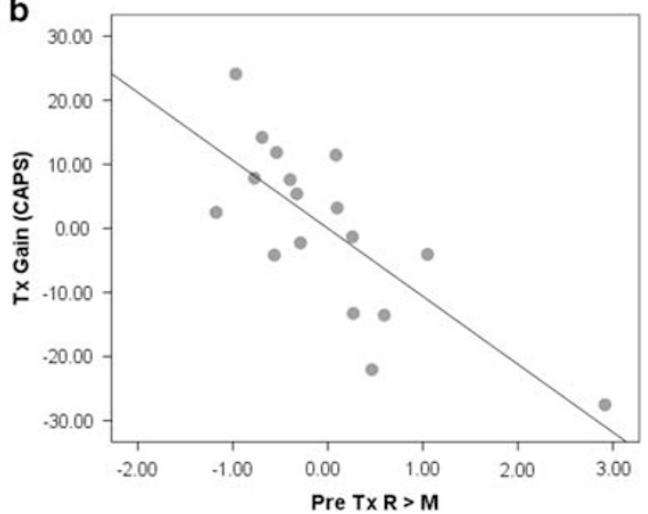

Figure 3 Pre-treatment Reappraise (>Maintain) activation predictive of treatment gain. (a) One-sample voxel-wise statistical t-map overlaid on a canonical brain rendering (Montreal Neurological Institute (MNI) sagittal), showing the location of the significant negative correlation between Pre-treatment right ventrolateral prefrontal cortex (VIPFC)/ inferior frontal gyrus (IFG) activation during Reappraise > Maintain (46, 44, - I0) and Pre-treatment > Post-treatment Clinician-Administered PTSD Scale (CAPS) scores, controlling for baseline symptom severity (ie, Pre-treatment CAPS scores) in the posttraumatic stress disorder (PTSD) group. Threshold for displaying the image is set at $p=0.05$ and masked; color bars represent statistical $t$-scores. (b) Scatterplot depicting the partial correlation between right vIPFC/IFG during Reappraise (> Maintain) and treatment-related symptom change (Pre-treatment - Post-treatment CAPS scores), controlling for baseline symptomatology (Pre-treatment CAPS scores). R>M, Reappraise > Maintain; Tx, Treatment.

executive aspects of motor control (Eickhoff et al, 2011) and has previously been implicated in emotion regulation success (Wager et al, 2008).

Results observed here are broadly in line with prior findings. For example, previous work in depressed patients found that SSRI treatment increased activation in the right dlPFC during an emotional interference task (Fales et al, 2009). In another study, depressed patients with greater symptom reduction over 6 months of SSRI treatment also showed greater increases in reappraisal-related dlPFC activation (Heller et al, 2013). In PTSD, prior work has been limited, with one resting state study showing that treatment with SSRIs increased activation in the left medial temporal cortex, whereas greater medial PFC activation post-treatment was associated with greater symptom change (Seedat et al, 2004). Another single-subject study found that a patient treated with SSRIs showed increased activation in the IFG, among other regions (Fernandez et al, 2001). Thus, although the present results differ somewhat from prior work in PTSD, when taken together with the literature on depression, the current and prior results suggest that SSRIs may exert their therapeutic effects via enhancement of activity in prefrontal regions known to be involved in the initiation and maintenance of emotion regulation (eg, Buhle et al, 2013; Kohn et al, 2014). Moreover, as shown here, SSRIs may also boost activation in other regions of the brain-ie, the SMA-believed to support the effective implementation of negative affect downregulation (Ray and Zald, 2012).

Other work has suggested that SSRIs may reduce activity in the amygdala in depression (Fu et al, 2004; Sheline et al, 2001) and certain anxiety disorders (Phan et al, 2013); however, such results were not observed here or in prior work in PTSD (Fernandez et al, 2001; Seedat et al, 2004). One possibility is that SSRIs may primarily exert their effects via the PFC in PTSD. Another possibility is that such results are observed particularly in relation to cognitive reappraisal. PTSD has been conceptualized as both a disorder of emotion regulation and as a fear disorder (eg, Ehlers and Clark, 2000; Foa et al, 1989). Therefore, future work may wish to use other tasks, such as fear conditioning and extinction paradigms, to determine whether additional neural correlates and predictors of response to SSRI treatment are revealed.

Lanius et al (2010) have distinguished between emotional undermodulation and emotional overmodulation in PTSD. Whereas emotional undermodulation is believed to involve hyperarousal and the reliving of traumatic experiences, and is characteristic of individuals with non-dissociative PTSD, emotional overmodulation is thought to involve emotional detachment, hypoarousal, and depersonalization, and may primarily characterize individuals with dissociative PTSD. Prior work suggests that emotional undermodulation involves reduced prefrontal activation and therefore reduced inhibition of limbic regions, whereas emotional overmodulation involves increased prefrontal activation and excessive inhibition of limbic regions (Nicholson et al, 2015). By finding that SSRI treatment increases prefrontal activation, the present results support an emotional undermodulation conceptualization of PTSD. Importantly, however, Lanius et al (2010) suggest that individuals with PTSD are best thought of as a heterogenous group rather than a single group. Therefore, future work may wish to examine how results would differ for different subtypes of PTSD (eg, dissociative versus non-dissociative).

This is the first study to identify fMRI predictors of SSRI treatment response in PTSD. A recent meta-analysis found that increased baseline activation in the insula and the striatum was predictive of poorer response to pharmacological or psychotherapeutic treatment in depression (Fu et al, 2004). In addition, one previous study found that anxious children (most with generalized anxiety disorder) who showed less baseline amygdala reactivity benefitted more from treatment with either SSRIs or psychotherapy (McClure et al, 2007). Thus, prior work is not directly comparable to the present study. The vlPFC/IFG has been implicated in inhibition and response control (for a review, see Bari and Robbins, 2013) and in emotion regulation (for a metaanalysis, see Buhle et al, 2013), and IFG activation during cognitive-affective tasks has been observed to be deficient in 
PTSD (for a meta-analysis, see Hayes et al, 2012). Therefore, initial deficits in this region might mean more room for improvement with SSRI treatment. Given the paucity of treatment prediction work in PTSD compared with other disorders, more work is needed as to how the neural correlates of SSRI treatment may differ in PTSD, anxiety, stressor-related disorders, and depression.

Limitations of this study include the lack of a placebocontrol condition for the PTSD group and an all-male, relatively small sample. We also studied only PTSD related to combat trauma and thus the findings cannot be generalized to PTSD from other types of trauma or to other anxiety disorders. Lastly, we focused on reappraisal-based emotion regulatory brain function and it remains unknown how the neural correlates and predictors of SSRI treatment relate to other forms of emotion regulation and/or emotion/fear reactivity in PTSD. Finally, the CEC and PTSD groups differed in age, education, and level of combat trauma exposure, which could have affected results in our post-hoc ANOVAs that examined Group $\times$ Time interactions in SMA and dlPFC $\mathrm{R}>\mathrm{M}$ activation.

In sum, SSRI treatment for PTSD enhanced activation in the left dlPFC and SMA during reappraisal, and less pre-treatment right vlPFC/IFG activation during reappraisal predicted greater treatment gains, irrespective of baseline symptom severity. Therefore, SSRIs may enhance reappraisalrelated activation in the PFC for patients with PTSD and patients with the greatest initial deficits in this region may stand to benefit the most from treatment. The results add to a growing literature seeking to understand treatment mechanisms and predictors in the service of developing new and improved interventions and to a clinical science aimed at developing a more personalized approach to treatment decision making. More work involving randomized assignment, comparative treatment, and transdiagnostic approaches are needed to further progress on these fronts.

\section{FUNDING AND DISCLOSURE}

This material is based on work supported by the Department of Veterans Affairs, Veterans Health Administration, Office of Research and Development, Clinical Sciences Research and Development, and the Veterans Affairs Merit Review Program Award, awarded to KLP. AM is supported by the National Institute of Mental Health grant T32MH067631-09. MBS has, in the past 3 years, received payment for his editorial work on the journals 'Depression and Anxiety' and 'Biological Psychiatry,' and on the evidence-based medical information source 'Up-To-Date'. He has also been paid as a consultant by Janssen, Pfizer, and Tonix Pharmaceuticals, and by Care Management Technologies. AM, CAR, AEK, DAF, IL and KLP have no potential conflicts of interest to declare.

\section{ACKNOWLEDGMENTS}

We thank Drs Mark Lyubkin and Brian Martis for their involvement in the clinical aspects of the treatment study. We acknowledge the OEF/OIF veterans for their participation in this research study and more importantly for their dedication and service to the United States.

\section{REFERENCES}

APA (2000). Diagnostic and Statistical Manual of Mental Disorders IV-TR. Amer Psychiatric Pub Inc.: Washington, DC.

Bari A, Robbins TW (2013). Inhibition and impulsivity: behavioral and neural basis of response control. Prog Neurobiol 108: 44-79.

Beck AT, Steer RA, Ball R, Ranieri W (1996). Comparison of Beck Depression Inventories-IA and -II in psychiatric outpatients. J Pers Assess 67: 588-597.

Berger W, Mendlowicz MV, Marques-Portella C, Kinrys G, Fontenelle LF, Marmar CR et al (2009). Pharmacologic alternatives to antidepressants in posttraumatic stress disorder: a systematic review. Prog Neuropsychopharmacol Biol Psychiatry 33: 169-180.

Blake DD, Weathers FW, Nagy LM, Kaloupek DG, Gusman FD, Charney DS et al (1995). The development of a clinicianadministered PTSD scale. J Trauma Stress 8: 75-90.

Blanchard EB, Jones-Alexander J, Buckley TC, Forenia CA (1996). Psychometric properties of the PTSD checklist (PCL). Behav Res Ther 34: 669-673.

Bremner JD, Staib LH, Kaloupek D, Southwick SM, Soufer R, Charney DS (1999). Neural correlates of exposure to traumatic pictures and sound in Vietnam combat veterans with and without posttraumatic stress disorder: a positron emission tomography study. Biol Psychiatry 45: 806-816.

Buhle JT, Silvers JA, Wager TD, Lopez R, Onyemekwu C, Kober H et al (2013). Cognitive reappraisal of emotion: a meta-analysis of human neuroimaging studies. Cereb Cortex 24: 2981-2990.

Ehlers A, Clark DM (2000). A cognitive model of posttraumatic stress disorder. Behav Res Ther 38: 319-345.

Eickhoff SB, Bzdok D, Laird AR, Roski C, Caspers S, Zilles K et al (2011). Co-activation patterns distinguish cortical modules, their connectivity and functional differentiation. NeuroImage 57: 938-949.

Fales CL, Barch DM, Rundle MM, Mintun MA, Mathews J, Snyder AZ et al (2009). Antidepressant treatment normalizes hypoactivity in dorsolateral prefrontal cortex during emotional interference processing in major depression. J Affect Disord 112: 206-211.

Fernandez M, Pissiota A, Frans O, Knorring L von, Fischer H, Fredrikson M (2001). Brain function in a patient with torture related post-traumatic stress disorder before and after fluoxetine treatment: a positron emission tomography provocation study. Neurosci Lett 297: 101-104.

First MB, Spitzer RL, Gibbon M, Williams JBW (2002). Structured Clinical Interview for DSM-IV Axis I Disorders, Research Version - Non-patient Edition (SCID-I/NP). Biometrics Research, New York State Psychiatric Institute: New York.

Foa EB, Steketee G, Rothbaum BO (1989). Behavioral/cognitive conceptualizations of post-traumatic stress disorder. Behav Ther 20: $155-176$.

Frank DW, Dewitt M, Hudgens-Haney M, Schaeffer DJ, Ball BH, Schwarz NF et al (2014). Emotion regulation: quantitative metaanalysis of functional activation and deactivation. Neurosci Biobehav Rev 45: 202-211.

Fu CHY, Williams SCR, Cleare AJ, Brammer MJ, Walsh ND, Kim J et al (2004). Attenuation of the neural response to sad faces in major depression by antidepressant treatment: a prospective, event-related functional magnetic resonance imaging study. Arch Gen Psychiatry 61: 877-889.

Ghashghaei HT, Hilgetag CC, Barbas H (2007). Sequence of information processing for emotions based on the anatomic dialogue between prefrontal cortex and amygdala. NeuroImage 34: 905-923.

Gross JJ (1998). Antecedent-and response-focused emotion regulation: divergent consequences for experience, expression, and physiology. J Pers Soc Psychol 74: 224-237.

Hamilton M (1960). A rating scale for depression. J Neurol Neurosurg Psychiatry 23: 56-62. 
Hayes JP, Hayes SM, Mikedis AM (2012). Quantitative metaanalysis of neural activity in posttraumatic stress disorder. Biol Mood Anxiety Disord 2: 9.

Heller AS, Johnstone T, Peterson MJ, Kolden GG, Kalin NH, Davidson RJ (2013). Increased prefrontal cortex activity during negative emotion regulation as a predictor of depression symptom severity trajectory over 6 months. JAMA Psychiatry 70: $1181-1189$.

Hoge CW, Castro CA, Messer SC, McGurk D, Cotting DI, Koffman RL (2004). Combat duty in Iraq and Afghanistan, mental health problems, and barriers to care. N Engl J Med 351: 13-22.

IOM NRC (2013). Returning Home from Iraq and Afghanistan: Assessment of Readjustment Needs of Veterans, Service Members, and Their Families. The National Academies Press: Washington, DC.

Keane TM, Fairbank JA, Caddell JM, Zimering RT, Taylor KL, Mora CA (1989). Clinical evaluation of a measure to assess combat exposure. Psychol Assess J Consult Clin Psychol 1: 53-55.

Kohn N, Eickhoff SB, Scheller M, Laird AR, Fox PT, Habel U (2014). Neural network of cognitive emotion regulation - An ALE metaanalysis and MACM analysis. NeuroImage 87: 345-355.

Lang PJ, Bradley MM, Cuthbert BN (2008). International affective picture system (IAPS): affective ratings of pictures and instruction manual. Technical Report A-8, University of Florida, Gainesville, FL.

Lanius RA, Vermetten E, Loewenstein RJ, Brand B, Schmahl C, Bremner JD et al (2010). Emotion modulation in PTSD: clinical and neurobiological evidence for a dissociative subtype. Am J Psychiatry 167: 640-647.

Ma Y (2014). Neuropsychological mechanism underlying antidepressant effect: a systematic meta-analysis. Mol Psychiatry 20: 311-319.

Maier W, Buller R, Philipp M, Heuser I (1988). The Hamilton Anxiety Scale: reliability, validity and sensitivity to change in anxiety and depressive disorders. J Affect Disord 14: 61-68.

McClure EB, Adler A, Monk CS, Cameron J, Smith S, Nelson EE et al (2007). fMRI predictors of treatment outcome in pediatric anxiety disorders. Psychopharmacology (Berl) 191: 97-105.

Miller EK, Cohen JD (2001). An integrative theory of prefrontal cortex function. Annu Rev Neurosci 24: 167-202.

Miller JM, Schneck N, Siegle GJ, Chen Y, Ogden RT, Kikuchi T et al (2013). fMRI response to negative words and SSRI treatment outcome in major depressive disorder: a preliminary study. Psychiatry Res Neuroimaging 214: 296-305.

New AS, Fan J, Murrough JW, Liu X, Liebman RE, Guise KG et al (2009). A functional magnetic resonance imaging study of deliberate emotion regulation in resilience and posttraumatic stress disorder. Biol Psychiatry 66: 656-664.

Nicholson AA, Densmore M, Frewen PA, Théberge J, Neufeld RWJ, McKinnon MC et al (2015). The dissociative subtype of posttraumatic stress disorder: unique resting-state functional connectivity of basolateral and centromedial amygdala complexes. Neuropsychopharmacology (doi:10.1038/npp.2015.79).

Ochsner KN, Bunge SA, Gross JJ, Gabrieli JDE (2002). Rethinking feelings: an fMRI study of the cognitive regulation of emotion. J Cogn Neurosci 14: 1215-1229.
Phan KL, Britton JC, Taylor SF, Fig LM, Liberzon I (2006). Corticolimbic blood flow during nontraumatic emotional processing in posttraumatic stress disorder. Arch Gen Psychiatry 63: 184-192.

Phan KL, Coccaro EF, Angstadt M, Kreger KJ, Mayberg HS, Liberzon I et al (2013). Corticolimbic brain reactivity to social signals of threat before and after sertraline treatment in generalized social phobia. Biol Psychiatry 73: 329-336.

Phan KL, Fitzgerald DA, Nathan PJ, Moore GJ, Uhde TW, Tancer ME (2005). Neural substrates for voluntary suppression of negative affect: a functional magnetic resonance imaging study. Biol Psychiatry 57: 210-219.

Rabinak CA, MacNamara A, Kennedy AE, Angstadt M, Stein MB, Liberzon I et al (2014). Focal and aberrant prefrontal engagement during emotion regulation in veterans with posttraumatic stress disorder. Depress Anxiety 31: 851-861.

Ray RD, Zald DH (2012). Anatomical insights into the interaction of emotion and cognition in the prefrontal cortex. Neurosci Biobehav Rev 36: 479-501.

Robert S, Hamner MB, Ulmer HG, Lorberbaum JP, Durkalski VL (2006). Open-label trial of escitalopram in the treatment of posttraumatic stress disorder. J Clin Psychiatry 67: 1522-1526.

Seedat S, Stein DJ, Ziervogel C, Middleton T, Kaminer D, Emsley RA et al (2002). Comparison of response to a selective serotonin reuptake inhibitor in children, adolescents, and adults with posttraumatic stress disorder. J Child Adolesc Psychopharmacol 12: $37-46$.

Seedat S, Warwick J, Heerden B, van, Hugo C, Zungu-Dirwayi N, Van Kradenburg J et al (2004). Single photon emission computed tomography in posttraumatic stress disorder before and after treatment with a selective serotonin reuptake inhibitor. J Affect Disord 80: 45-53.

Sheline YI, Barch DM, Donnelly JM, Ollinger JM, Snyder AZ, Mintun MA (2001). Increased amygdala response to masked emotional faces in depressed subjects resolves with antidepressant treatment: an fMRI study. Biol Psychiatry 50: 651-658.

Shin LM, Orr SP, Carson MA, Rauch SL, Macklin ML, Lasko NB et al (2004). Regional cerebral blood flow in the amygdala and medial prefrontal cortex during traumatic imagery in male and female Vietnam veterans with PTSD. Arch Gen Psychiatry 61: 168-176.

Simmons AN, Matthews SC (2012). Neural circuitry of PTSD with or without mild traumatic brain injury: a meta-analysis. Neuropharmacology 62: 598-606.

Tzourio-Mazoyer N, Landeau B, Papathanassiou D, Crivello F, Etard O, Delcroix N et al (2002). Automated anatomical labeling of activations in SPM using a macroscopic anatomical parcellation of the MNI MRI single-subject brain. Neuroimage 15: 273-289.

Vermetten E, Schmahl C, Southwick SM, Bremner JD (2007). A positron tomographic emission study of olfactory induced emotional recall in veterans with and without combat-related posttraumatic stress disorder. Psychopharmacol Bull 40: 8-30.

Wager TD, Davidson ML, Hughes BL, Lindquist MA, Ochsner KN (2008). Prefrontal-subcortical pathways mediating successful emotion regulation. Neuron 59: 1037-1050.

Supplementary Information accompanies the paper on the Neuropsychopharmacology website (http://www.nature.com/npp) 